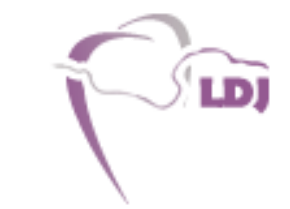

\title{
ORAL VERRUCOUS CARCINOMA: A REPORT OF THREE CASES
}

Smit Singla ${ }^{1}$, Raghavendra Kini², Vathsala Naik², Anjali Shetty²

1) Department Of Oral Medicine \& Radiology, Teerthanker Mahaveer Dental College, Moradabad, India

2) Department of Oral Medicine \& Radiology, A.J. Institute of Dental Sciences, Mangalore, India

\section{ARTICLE INFORMATION:}

\section{Article History:}

Received: 6 January, 2014

Accepted in revised form: 1February

2014

Published: 8 July 2014

Corresponding author:

Smit Singla

E-mail: smitcool2@yahoo.co.in

\section{Keyword:}

Verrucous carcinoma, buccal

mucosa, alveolar mucosa

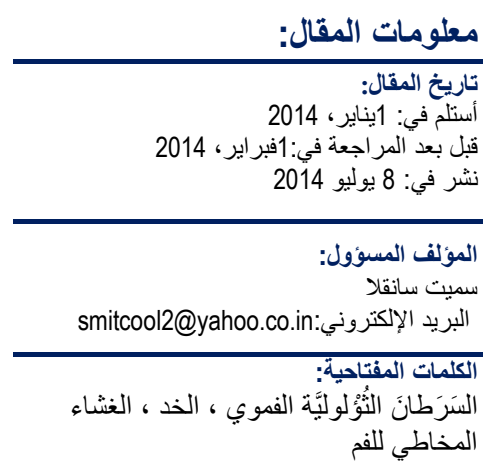

\begin{abstract}
:
Ackerman's tumor is a low grade variant of oral Verrucous carcinoma (VC) or squamous cell carcinoma (OSCC). Although it occurs in other anatomic sites, most intraoral cases involve buccal mucosa, alveolar mucosa and gingiva. The typical features of $\mathrm{VC}$ that differ from the usual oral epidermoid carcinoma is it generally slow growing, chiefly exophytic and superficially invasive at until late in the course of the disease and have low metastatic potential. This paper presents three variable cases of oral VC along with their clinical and histo-pathological features.
\end{abstract}

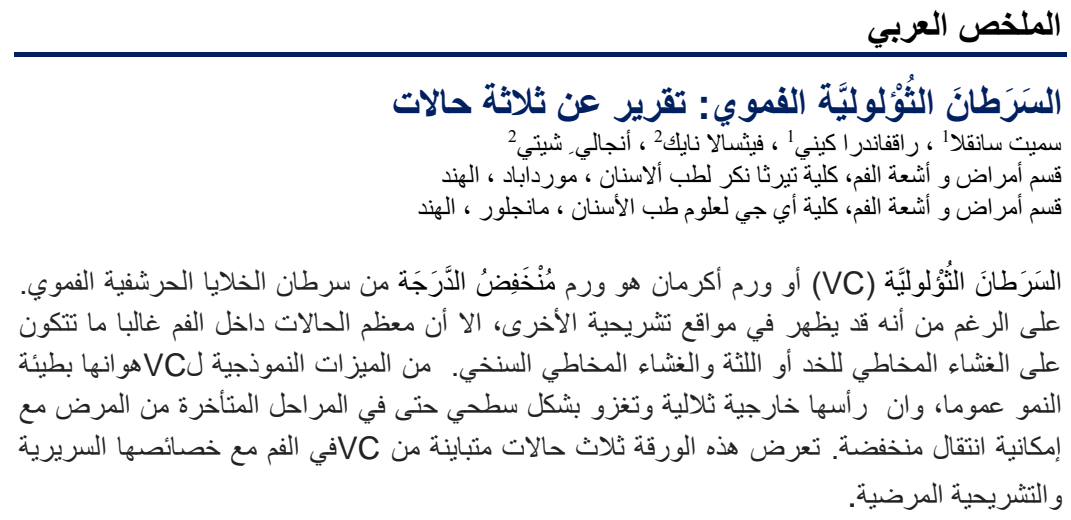




\section{INTRODUCTION:}

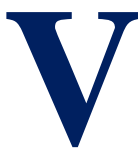

errucous carcinoma is a variant of squamous cell carcinoma that can affect cutaneous and mucosal surfaces. Different terms are used to describe this tumor, including Ackerman's tumor, Buschke Loe wenstein tumor, florid oral papillomatosis, epithelial cuniculatum and carcinoma cuniculatum $^{1,2}$. The ages range from 50 to 80 years with a male predominance and the median age is 67 years ${ }^{3,4}$. VC may grow very large and can destroy adjacent tissue such as bone and cartilage ${ }^{5}$.Clinical feature depends on several factors such as duration of lesion, degree of keratinization along with changes in adjacent mucosa. The developed carcinoma shows an exophytic gray to red bulky lesion with a rough, shaggy, papillomatous surface. The term 'verrucous' is used because of its fine, finger like surface projections ${ }^{6,7}$. It may grow into soft tissue and invade bone, regional lymph nodes are usually tender but metastasis is rare. It is usually associated with chronic exposure to UVrays, prolonged use of tobacco and its products including betel nut and snuff. It is also called "snuff dipper's cancer". The oncogenic viruses HPV 16 and 18 are also implicated in the etiology $\mathrm{y}^{8}$.

\section{CASE SERIES:}

\section{Case-1}

A 58 years old male patient reported to the department with a chief complaint of pain on left cheek since 4 months. Patient noticed it 4 months back, pain was intermittent, moderate in intensity and does not vary with postural variation. Pain was associated with ulcer since two months. No history of discharge and paresthesia. Patient used to chew mixture of areca nut, tobacco and lime 8-10 times from last 30 years. On intraoral examination an exophytic ulcer proliferative growth was present on left buccal mucosa measuring about $6 \times 2 \mathrm{~cm}$ extending from commissural area to retro molar area at the line of occlusion. Overlying mucosal surface was rough, irregular covered with slough. Erythematous pinpoints was inter dispersed throughout the lesion with rolled out edges,Fig1. Basic findings on palpation, differential diagnosis and procedure (biopsy and surgical excision) done were the same for cases 1 and 2 (as discussed below).

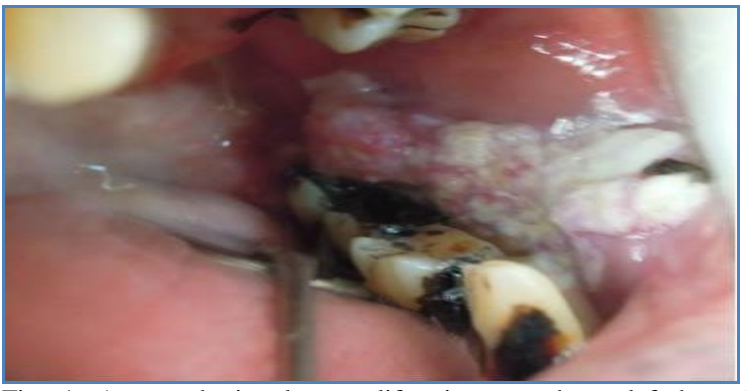

Fig: 1. An exophytic ulcer proliferative growth on left buccal mucosa extending from commissural area to retro molar area at the line of occlusion.

Surgical excision was done with including normal surrounding mucosa and reconstruction with collagen membrane graft was done, Fig2.

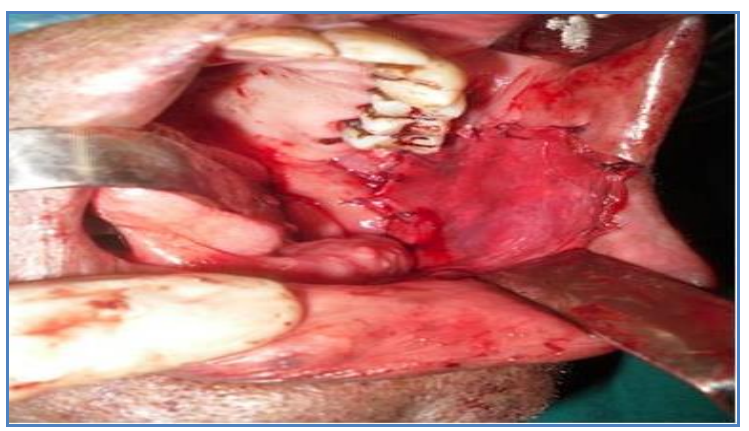

Fig:2. post-operative after reconstruction with collagen membrane graft

\section{Case-2}

A female patient aged 60 years, reported with a chief complaint of pain on right cheek since 6 months. Patient noticed it 6 months back, pain was intermittent, moderate in intensity and does not vary with postural variation. Pain was associated with ulcer since six months. No history of discharge and paresthesia. Patient used to chew mixture of areca nut, tobacco and lime 6-8 times from last 20 years. On intraoral examination an exophytic ulcer proliferative growth was present on right buccal mucosa measuring about $6 \times 5 \mathrm{~cm}$ extending superiorinferiorly from lower right buccal vestibule to upper right buccal vestibule and extending to the right maxillary edentulous ridge and anterioposteriorly from right commissural area involving the lower lip to retromolar area. Overlying mucosal surface was rough, irregular covered with slough. Erythematous pinpoints was inter dispersed throughout the lesion with rolled out edges, Fig3. 


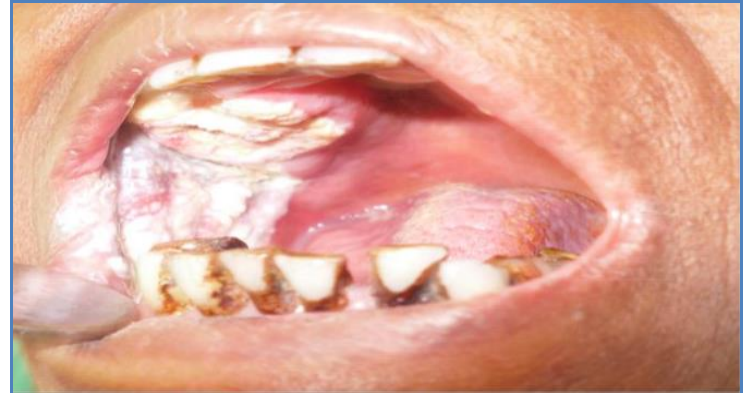

Fig: 3. exophyticulcero proliferative growth on right buccal mucosa

On palpation all relevant inspectory finding were confirmed. Ulcero-proliferative growth was tender on palpation, firm in consistency and ulcer was indurated at the margins. Surface was irregular with well-defined edges. Right submandibular lymph nodes were palpable two in number, mobile, firm in consistency and nontender. Based on the history and clinical findings provisional diagnosis of verrucous carcinoma of left buccal mucosa was made. Squamous cell carcinoma and metastatic carcinoma were considered underdifferential diagnosis. Incisional biopsy was done and revealed loss of parakeratin is projecting into rete ridges and suggestive of verrucous carcinoma Fig4.

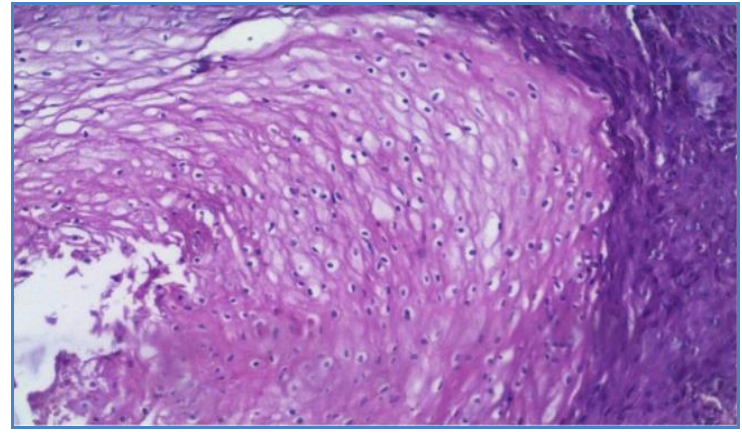

Fig: 4. histological conformation of verrucous carcinoma

Surgical excision was done with including normal surrounding mucosa and reconstruction with collagen membrane graft was done, Fig5.

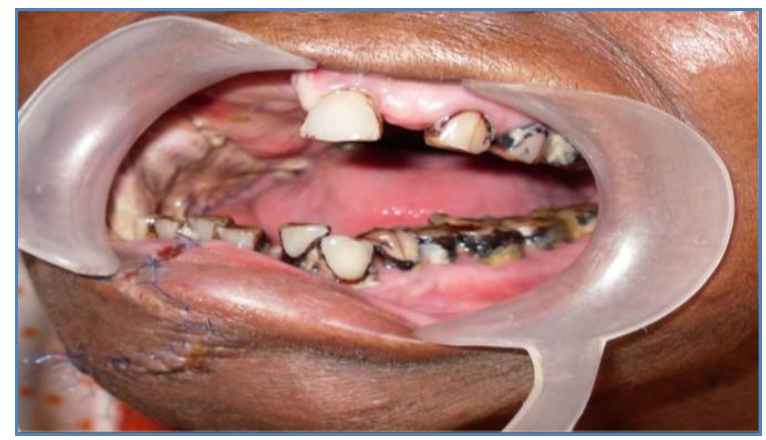

Fig: 5. post-operative after reconstruction with collagen membrane graft

Citation:Libyan Dent J 2014, 4:17317742 - http://dx.doi.org/10.5542/LDJ.v4i0. 17317742

\section{Case-3}

A 65 year old female patient reported to the department with a chief complaint of pain on the left cheek since last 4 months. Patient noticed it 4 months back. Pain was intermittent, moderate in intensity and does not vary with postural variation. Pain was localized and according to patient pain was due to crack on lip and not aware of growth on the buccal mucosa. Pain increased while eating and decreased after eating. Past medical and dental histories were noncontributory. Patient used to chew areca nut, tobacco, slaked lime 5 to 6 times daily from last 20 years. Patient used to chew and used to keep in the oral cavity for around 15-20 minutes. After keeping 15-20 minute patient used to spit the stuff. An exophytic ulcero-proliferative growth was present on left buccal mucosa measuring about $3 \times 2 \mathrm{~cm}$. extending from commissural area at the line of occlusion, Fig6.

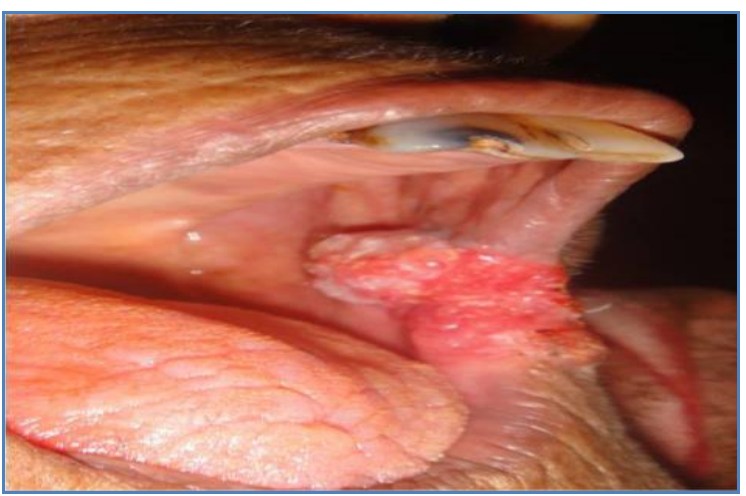

Fig:6.exophyticulcero-proliferative growth on left buccal mucosa extending from commissural area at the line of occlusion

Overlying surface is rough covered with slough having erythematous pinpoints with rolled out edges. On palpation lesion was firm in consistency with indurations at the margins and does not bleed on palpation. Surface was irregular with well- defined edges. Based on clinical examination carcinoma of left buccal mucosa was given. Verrucous carcinoma was considered under differential diagnosis. Incisional biopsy was done and revealed parakeratin plugging, rete ridges are bulbous, epithelial margin appears to be pushing into the connective tissue with intact basement membrane and suggestive of Verrucous carcinoma. Surgical excision of the lesion with surrounding tissue was done. Follow up of one year was done for all the three cases till that there was no recurrence. 


\section{DISCUSSION:}

Due to its benign indolent behavior, most of the time it will go unrecognized or unchallenged by the patient. Earlier lesion starts as verrucous hyperplasia, later it become vegetant and clinically resembles verrucous leukoplakia. Evolution of verrucous carcinoma from primary lesion takes months to years ${ }^{9}$. It occurs in elderly patient around 60 to 70 years of age similar to our case series. It is more common in men as compared to women whereas we found 2 cases in women out of 3 cases ${ }^{3,4}$.Verrucous carcinoma constitutes 2 to $4.5 \%$ of all forms of squamous cell carcinoma in the oral cavity ${ }^{6}$.Verrucous is usually associated with the use of tobacco and its products as seen in our all the three cases. Clinical features vary depends upon the duration and keratinization of the lesion. Typically lesion is a pale, warty, fungating, locally aggressive, ulcerated tumor attached by a broad base, is well circumscribed and it is clearly demarcated from the adjacent mucosa. Tumor has a predominant horizontal growth, it tend to erode more than infilterate $^{10}$. Regional lymph node metastases are exceedingly rare. Ferlito et al. (1980) emphasized on the following classic description for the diagnosis of verrucous carcinoma: a) Fungating warty tumor, b)Thickened club shaped, papillomatous projections which push rather than infiltrate into the underlying tissue, c) Deeply projecting cleft like spaces with degenerating keratin and later cystic degeneration of central portion of the filiform projections, d)High degree of cellular Differentiation with absence of features of malignancy, e)Considerable inflammatory response in invaded tissues, f)Rare regional lymph node and distant metastasis ${ }^{11}$. There are four clinic pathologic types: (1) an urogenital: giant condyloma acuminatum, Buschke-Loewenstein tumor, giant malignant condyloma, verrucous carcinoma of the anogenital mucosa, carcinoma-like condyloma, and condylomatoid Precancerosis, (2) oro-aerodigestive: Ackerman tumor, verrucous carcinoma of Ackerman, oral florid papillomatosis, (3) Feet: epithelioma cuniculatum, carcinoma cuniculatum, (4) Other cutaneous sites: cutaneous verrucous carcinoma, papillomatosis cutis carcinoides, papillomatosis cutis $^{12}$. Buccal mucosa is most commonly affected site followed by mandibular retromolar and molar area (41.6\%) followed by the buccal mucosa. (16.6\%), the hard palate (16.6\%), the floor of the mouth (16.6\%), and the lip mucosa $(8.3 \%)^{2}$. Microscopically, verrucous carcinomas consist of thickened club shaped filiform projections lined with thick, well-differentiated squamous epithelium with marked surface keratinisation ("church-spire" keratosis). Parakeratin typically fills the numerous clefts or crypts (parakeratin plugs) between the surface projections as was seen in our case reports. Clinically verrucous hyperplasia and verrucous carcinoma are indistinguishable. In 10 to $20 \%$ cases following irradiation biological behavior of small proportion of verrucous carcinoma are reported within extremely short latent period, called anaplastic transformation. Some authors don't believe in this 'dedifferentiation' phenomenon and account this observation due to presence of 'hybrid tumors', i.e. presence of foci of less differentiated squamous cell carcinoma within verrucous carcinoma ${ }^{6}$. Management of oral verrucous carcinoma include adequate oral hygiene and surgical excision of the lesion with adequate margins is the treatment of the choice and prognosis is good as done in our all the cases. Surgical excision can be done with laser which provides the bloodless field. If cervical lymph nodes were palpable then ultra sound guided fine needle aspiration biopsy were advised, in our cases there was no cervical lymphadenopathy. Depending upon the lymph node metastasis supraomohyoid or redundant neck dissection can be done ${ }^{15}$. Irradiation alone or in combination with surgery is rarely performed. Combined therapy can be useful when the tumor extends to the retromolar area. When surgery is not indicated, other treatment modalities such as cytostatic drugs may be preferred. Various dosages of cytostatic drugs have been proven to show beneficial effects in reducing tumor size; $\alpha$-interferon (IFN) seems to support the therapy by delaying the growth of the tumor but does not take the place of surgery alone ${ }^{2}$. "No matter what the treatment is, the rate of local recurrences is said to be high ranging from $30 \%$ to $50 \%$ and not unusually is the result of inadequate surgery because of the size of the tumor and left dysplasia close to the verrucous carcinoma" $"$.

\section{CONCLUSION:}

Verrucous carcinomais frequently misdiagnosed. So, it is the need of the hour for the cooperation 
between clinician and pathologist to prevent the misdiagnosis. So that verrucous carcinoma should be diagnosed at the early stage to prevent the effect on the quality of life of the patients ${ }^{14}$.Different modes of treatment modalities are present based on the different presentations of the lesion.

\section{REFERENCES:}

1) Schwartz RA. Verrucous carcinoma of the skin and mucosa. J Am Acad Dermatol.1995 Jan;32(1):1-21; quiz 22-4. Review. Erratum in: J Am Acad Dermatol 1995May;32(5 Pt 1):710

2) Alkan A, Bulut E, Gunhan O, Ozden B. Oral verrucous carcinoma: a study of 12 cases. Eur J Dent. 2010 Apr;4(2):202-7.

3) Kang CJ, Chang JT, Chen TM, Chen IH, Liao CT. Surgical treatment of oral verrucous carcinoma. Chang Gung Med J. 2003 Nov;26(11):807-12.

4) Tornes K, Bang G, Strømme Koppang H, Pedersen KN. Oral verrucous carcinoma. Int J Oral Surg. 1985 Dec;14(6):485-92.

5) Koch BB, Trask DK, Hoffman HT, Karnell LH, Robinson RA, Zhen W, Menck HR; Commission on Cancer, American College of Surgeons; American Cancer Society. National survey of head and neck verrucous carcinoma: patterns of presentation, care, and outcome. Cancer. 2001 Jul 1;92(1):110-20.

6) Singh K, Kalsotra P, Khajuria R and Manhas M: Verrucous carcinoma (Ackerman"s Tumor) of mobile tongue. JK Science 2004; 6(4): 220222.
7) Mehta FS, Hammer JE. Tobacco related, oral mucosal lesion and conditions in India. Publication: Basic dental research unit. TIFR 1983; 3: 4.

8) Padmavathy L. et al. Verrucous Carcinoma report on two cases. Indian J Dermatol 2009; 54:S68-70.

9) R.B.V. Kumar, A.C. Raj Verrucous carcinoma of lip- an unusual presentation. Amrita Journal of Medicine 2012;8(2):40-42.

10) Varshney S, Singh J, Saxena RK, Kaushal A, Pathak VP. Verrucous carcinoma of larynx. Indian J Otolaryngol Head Neck Surg(2004); 56(1): 54-56.

11) Indudharan $R$, Das $P K$, Thida $T$. Verrucous carcinoma of maxillary antrum. Singapore Med J (1996); 37: 559-561.

12) Steffen C. The man behind the eponym: Lauren V. Ackerman and verrucous carcinoma of Ackerman. Am J Dermatopathol. 2004 Aug;26(4):334-41.

13) Depprich R.A, Handschel J.G, Fritzemeier C.U, Engers $\mathrm{R}$ and Kubler N.R: Hybrid verrucous carcinoma of the oral cavity: A challenge for the clinician and the pathologists. Oral Oncol Extra; 2006; 42: 85-90.

14) $\mathrm{N}$ Kaushal and $\mathrm{N}$ Madan, Verrucours carcinoma of the oral cavity: Case report, The Internet Journal of Geriatrics and Gerontology, 2011; 6(1).

15) M Kawakami, K Yoshimura, I Hayashi, K Ito, S Hyo. Verrucous Carcinoma of the Tongue: Report of two cases. Bulletin of the Osaka Medical College 2004;50:19-22 